\title{
Molecular Confirmation of Rocky Mountain Spotted Fever Epidemic Agent in Mexicali, Mexico
}

\section{Luis Tinoco-Gracia, Moisés Rodríguez Lomelí, Sawako Hori-Oshima, Nicole Stephenson, Janet Foley}

Since 2008, a large epidemic of Rocky Mountain spotted fever has been emerging among humans and dogs in Mexicali, adjacent to the United States in Baja California, Mexico. We molecularly confirmed the causative agent; this information can be used to study the origin and dynamics of the epidemic.

$\mathrm{R}$ ocky Mountain spotted fever (RMSF), caused by the bacteria Rickettsia rickettsii, is responsible for more human deaths than any other tickborne disease in North America (1). During 1999-2007, a total of 80 fatal cases were reported from Sonora, Mexico, alone (2). Recent epidemics in Arizona (USA) and Sonora have been associated with the brown dog tick (Rhipicephalus sanguineus) $(3,4)$, whereas most cases in the United States have been transmitted by bites of infected Dermacentor spp. ticks (5). The risk to humans is heightened by the epidemiologic cycle of the brown dog tick, a cosmopolitan tick that prefers the dog as its host and can live its entire life cycle in a periurban setting, often spending its off-host time indoors. $R$. sanguineus ticks, in addition to being vectors of $R$. rickettsii, are probable or confirmed vectors of Leishmania, Coxiella burnetii, and $R$. conorii (6).

\section{The Study}

In 2008, an epidemic of RMSF began in Mexicali, adjacent to the US border in Baja California, Mexico. In 2015, the Mexican Ministry of Health declared the epidemic an epidemiologic emergency, which as of 2018 has affected $\approx 4,000$ persons. In 2014 , a fatal human case in Imperial County, CA, USA, was probably associated with the Mexicali epidemic. Overall, since 2000, in the United States, the incidence of RMSF has reportedly increased $\approx 4$-fold (7); this dramatic increase may be caused in part by increased transmission via the brown dog tick but also by

Author affiliations: Universidad Autónoma de Baja California, Mexicali, Mexico (L. Tinoco-Gracia, M. Rodríguez Lomelí,

S. Hori-Oshima); University of California, Davis, California, USA

(N. Stephenson, J. Foley)

DOI: https://doi.org/10.3201/eid2409.171523 changes in reporting and inclusion of false-positive test results in case diagnoses.

Local response to the ongoing epidemic in Mexicali has involved the Secretariat of Health and doctors and researchers at the Universidad Autónoma de Baja California schools of medicine and veterinary medicine. During 2008-2009, in the impoverished neighborhood of Los Santorales in Mexicali, at least 13 persons died of RMSF. Under agreement with the Sector Salud de Mexicali, the Universidad Autónoma de Baja California veterinary team documented $81 \%$ seroprevalence among local dogs and confirmed active $R$. rickettsii infection in a human resident by conducting PCR of kidney tissue (8). Of 120 persons from Mexicali with clinical signs compatible with RMSF, 30 were positive by PCR for the gene gltA, according to an unpublished method (9). In 2014, the local team partnered with researchers at the University of California, Davis (Davis, California, USA), to further molecularly characterize the strains of $R$. rickettsii and $R$. sanguineus ticks from Mexicali. We provide definitive molecular confirmation of the identity of the disease agent causing the Mexicali epidemic.

The University of California, Davis, laboratory received DNA extracted by use of QIAGEN Blood and Tissue Kits (Valencia, CA, USA) from 16 cases from Mexico. Initial $R$. rickettsia-specific real-time PCR for the citrate synthase gene (10) was positive for 10 samples. To obtain products for DNA sequencing, we performed traditional PCR for the ompA and $17 \mathrm{kDa}$ genes as published $(11,12)$. Sequence-confirmed positive DNA and water-containing negative control reactions were incorporated in each PCR run. Results were assessed by electrophoresis and UVtransillumination of 1\% agarose gels stained with Gelstar (Lonza, Rockland, ME, USA). Bands of the expected size were excised and cleaned with a QIAquick Gel Extraction Kit (QIAGEN) according to the manufacturer's instructions. Products were sequenced in the forward and reverse directions in an ABI Prism 3730 Genetic Analyzer at the ${ }^{\mathrm{UC}}$ DNA Sequencing Facility at the University of California, Davis. Sequences were manually trimmed and corrected if the nucleotide could be unambiguously determined, then aligned by using CLC Main Workbench 6 (CLC bio, Waltham, MA, USA).

We successfully obtained ompA and $17 \mathrm{kDa}$ products from 5 samples and compared the sequences with those in 
the GenBank database by using BLAST (http://blast.ncbi. nlm.nih.gov/Blast.cgi). For ompA, the resulting 472-bp amplicons from the 5 products from Mexicali were $100 \%$ similar. For this gene, numerous accessions in GenBank also have $100 \%$ homology with $100 \%$ coverage, including strains Sheila Smith, Hauke, Hilo, Colombia, and Arizona. Sequences of $17 \mathrm{kDa}$ spanned $206 \mathrm{bps}$ and were also completely homologous among them. This gene did not differentiate to species but was $100 \%$ homologous with $R$. rickettsii, $R$. parkeri, and others in the database. Representative sequences from Mexicali were submitted to GenBank (accession nos. KY689935 for ompA and KY824575 for $17 \mathrm{kDa}$ ).

Among sequence-confirmed samples, data were not available for 1 sample. The other 4 samples were collected in June, July, and September 2013 and April 2014. Two samples were from men (41 and 25 years of age) and 2 from women (18 and 29 years of age); all patients had dogs with ticks. Signs and symptoms were fever and headache for all; for 1 patient, a rash and convulsions also developed. The 2 men died and the 2 women survived with treatment. All patients had home addresses in various parts of Mexicali, including central west, southwest, and southeast bordering agricultural land. Clinical data were not available for patients for whom samples were considered PCR positive but not sequence confirmed, although inclusion of such clinical data and risk factors could bias interpretation if they were false positive or only weakly positive.

\section{Conclusions}

The RMSF epidemic in Mexicali has not been contained and may be spreading to other parts of Baja California and into the United States. More data are needed before we can understand why this epidemic emerged, where the specific areas of high risk for exposure to infected ticks are located, and whether the particular $R$. rickettsii strain or relationship with this $R$. sanguineus tick strain is likely to be particularly invasive or virulent. Pockets of RMSF have occurred in Mexico since at least 1947, when cases attributable to the brown dog tick in Sonora, Sinaloa, Coahuila, and Durango were described (13). Given the very limited phylogeographic resolution available for $R$. rickettsii in many of the commonly used PCR products (14), it is not known whether the bacteria in the Mexicali epidemic originated from Sonora or more distantly. Next steps include obtaining a culture of the bacteria from Mexicali, studying bacterial virulence in vitro or in animal models, and assessing vector competence of the Mexicali $R$. sanguineus tick strain for $R$. rickettsii. Epidemiologic data on the spatial distribution and prevalence of infection in dogs are needed.

Aggressive intervention achieved partial and temporary resolution of the Arizona and Sonora epidemics, which were localized and relatively small; these interventions included dog spay and neuter programs, treatment of houses against ticks, and use of a long-acting tick collar (Seresto; Bayer, Shawnee Mission, KS, USA) directly on the dogs (15). However, the dog collars were initially donated and are prohibitively expensive and not feasible for the scope of the Mexicali epidemic. This large epidemic in a major city will require a far greater and more creative public health response. Studying this epidemic offers an opportunity to understand the origin and dynamics of this epidemic and can inform response to emerging tickborne diseases in general.

\section{Acknowledgments}

We thank Zachary Villareal for laboratory support and Marian Fierro, Christopher Paddock, William Nicholson, and Michael Levin for logistical and scientific support.

Financial support was provided by the University of California Institute for Mexico and the United States.

\section{About the Author}

Dr. Tinoco-Gracia is a research professor in the School of Veterinary Medicine at Universidad Autonoma de Baja California and director of the Laboratory of Veterinary Public Health Sciences. He studies zoonotic diseases of dogs and humans in Mexicali and leads community education programs of Universidad Autónoma de Baja California.

\section{References}

1. Biggs HM, Behravesh CB, Bradley KK, Dahlgren FS, Drexler NA, Dumler JS, et al. Diagnosis and management of tickborne rickettsial diseases: Rocky Mountain spotted fever and other spotted fever group rickettsioses, ehrlichioses, and anaplasmosisUnited States. MMWR Recomm Rep. 2016;65(RR-1):1-44. http://dx.doi.org/10.15585/mmwr.rr6502a1

2. Álvarez-Hernández G, Roldán JFG, Milan NSH, Lash RR, Behravesh CB, Paddock CD. Rocky Mountain spotted fever in Mexico: past, present, and future. Lancet Infect Dis. 2017;17:e18996. http://dx.doi.org/10.1016/S1473-3099(17)30173-1

3. Nicholson WL, Paddock CD, Demma L, Traeger M, Johnson B, Dickson J, et al. Rocky Mountain spotted fever in Arizona: documentation of heavy environmental infestations of Rhipicephalus sanguineus at an endemic site. Ann N Y Acad Sci. 2006;1078:338-41. http://dx.doi.org/10.1196/annals.1374.065

4. Álvarez-Hernández G. La fiebre manchada de las Montañas Rocosas, una epidemia olvidada. Salud Pública de México. 2010;52:1-3.

5. Regan JJ, Traeger MS, Humpherys D, Mahoney DL, Martinez M, Emerson GL, et al. Risk factors for fatal outcome from Rocky Mountain spotted fever in a highly endemic area-Arizona, 20022011. Clin Infect Dis. 2015;60:1659-66. http://dx.doi.org/10.1093/ cid/civ116

6. Dantas-Torres F. Biology and ecology of the brown dog tick, Rhipicephalus sanguineus. Parasit Vectors. 2010;3:26. http://dx.doi.org/10.1186/1756-3305-3-26

7. Centers for Disease Control and Prevention. Rocky Mountain spotted fever-statistics and epidemiology [cited 2017 Aug 1]. https://www.cdc.gov/rmsf/stats/index.html 
8. Tinoco L. Diagnostico serologico y molecular de un brote de riquetsiosis (Rickettsia rickettsii) en perros y un humano en la cuidad de Mexicali, B.C. XXXIV Congreso Nacional de Infectologia, México; 2009 Oct 7-10; Guadalajara, Jalisco State, Mexico.

9. Gómez-Castellanos P, Tinoco-Gracia L, López-Valencia G, Oshima S, Medina Basulto G. Deteccion de especies del genero Rickettsia por PCR en pacientes del sector salud y analisis de factores de riesgo en Mexicali Baja California. Rev Biomed. $2015 ; 26: 59$.

10. Kato CY, Chung IH, Robinson LK, Austin AL, Dasch GA, Massung RF. Assessment of real-time PCR assay for detection of Rickettsia spp. and Rickettsia rickettsii in banked clinical samples. J Clin Microbiol. 2013;51:314-7. http://dx.doi.org/10.1128/ JCM.01723-12

11. Anstead CA, Chilton NB. A novel Rickettsia species detected in vole ticks (Ixodes angustus) from western Canada. Appl Environ Microbiol. 2013;79:7583-9. http://dx.doi.org/10.1128/AEM.02286-13
12. Webb L, Carl M, Malloy DC, Dasch GA, Azad AF. Detection of murine typhus infection in fleas by using the polymerase chain reaction. J Clin Microbiol. 1990;28:530-4.

13. Bustamante M, Varela G. Distribucion de las rickettsiasis en Mexico. Rev Inst Salubr Enferm Trop. 1947;8:3-13.

14. Paddock CD, Denison AM, Lash RR, Liu L, Bollweg BC, Dahlgren FS, et al. Phylogeography of Rickettsia rickettsii genotypes associated with fatal Rocky Mountain spotted fever. Am J Trop Med Hyg. 2014;91:589-97. http://dx.doi.org/10.4269/ajtmh.14-0146

15. Drexler N, Miller M, Gerding J, Todd S, Adams L, Dahlgren FS, et al. Community-based control of the brown dog tick in a region with high rates of Rocky Mountain spotted fever, 2012-2013. PLoS One. 2014;9:e112368. http://dx.doi.org/10.1371/journal.pone.0112368

Address for correspondence: Janet Foley, University of California, Department of Medicine and Epidemiology, 1320 Tupper Hall, Davis, CA 95616, USA; email: jefoley@ucdavis.edu

\section{July 2013: Vectorborne Infections}

- Transmission of Streptococcus equi Subspecies zooepidemicus Infection from Horses to Humans

- Travel-associated IIIness Trends and Clusters, 2000-2010

- Quantifying Effect of Geographic Location on Epidemiology of Plasmodium vivax Malaria
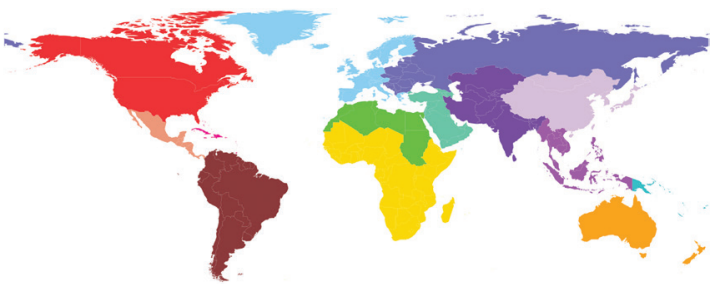

- Mutation in Spike Protein Cleavage Site and Pathogenesis of Feline Coronavirus

- Pneumococcal Serotypes before and after Introduction of Conjugate Vaccines, United States, 1999-2011

- Influence of Pneumococcal Vaccines and Respiratory Syncytial Virus on Alveolar Pneumonia, Israel

- Avian Metapneumovirus Subgroup C Infection in Chickens, China

- Human Alveolar Echinococcosis in Kyrgyzstan

- Molecular Epidemiologic Source Tracking of Orally Transmitted Chagas Disease, Venezuela

- Unique Clone of Coxiella burnetii Causing Severe Q Fever, French Guiana

- Babesia microti Infection, Eastern Pennsylvania, USA

- Reemergence of Chikungunya Virus in Bo, Sierra Leone

- Novel Bartonella Agent as Cause of Verruga Peruana
- Schmallenberg Virus among Female Lambs, Belgium, 2012

- Psychrobacter arenosus Bacteremia after Blood Transfusion, France

- Ciprofloxacin-Resistant Campylobacter spp. in Retail Chicken, Western Canada

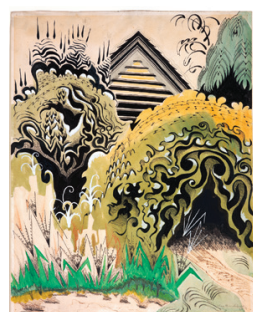

- Asynchronous Onset of Clinical Disease in BSE-Infected Macaques

- Prevalence of Nontuberculous Mycobacteria in Cystic Fibrosis Clinics, United Kingdom, 2009

- Reducing Visceral Leishmaniasis by Insecticide Impregnation of Bed-Nets, Bangladesh

- Genetic Variants of Orientia tsutsugamushi in Domestic Rodents, Northern China

- Undetected Multidrug-Resistant Tuberculosis Amplified by First-line Therapy in Mixed Infection

- Clinical Findings for Early Human Cases of Influenza A(H7N9) Virus Infection, Shanghai, China

- Multidrug-Resistant Atypical Variants of Shigella flexneri in China

- MDR TB Transmission, Singapore

- Human Infection with Marten Tapeworm

- Novel Bat-borne Hantavirus, Vietnam

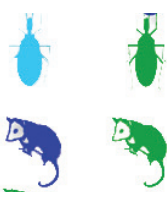

